\title{
A Control Method for Joint Torque Minimization of Redundant Manipulators Handling Large External Forces
}

\author{
Jon Woolfrey ${ }^{1}$ (D) $\cdot$ Wenjie Lu ${ }^{1} \cdot$ Dikai Liu $^{1}$
}

Received: 15 March 2018 / Accepted: 29 November 2018 / Published online: 11 January 2019

(C) The Author(s) 2019

\begin{abstract}
In this paper, a control method is developed for minimizing joint torque on a redundant manipulator where an external force acts on the end-effector. Using null space control, the redundant task is designed to minimize the torque required to oppose the external force, and reduce the dynamic torque. Furthermore, the joint motion can be weighted to factor in physical constraints such as joint limits, collision avoidance, etc. Conventional methods for joint torque minimization only consider the internal dynamics of the manipulator. If external forces acting on the end-effector are inadvertently implemented in to these control methods this could lead to joint configurations that amplify the resulting joint torque. The proposed control method is verified through two different case studies. The first case study involves simulation of high-pressure blasting. The second is a simulation of a manipulator lifting and moving a heavy object. The results show that the proposed control method reduces overall joint torque compared to conventional methods. Furthermore, the joint torque is minimized such that there is potential for a manipulator to execute certain tasks beyond its nominal payload capacity.
\end{abstract}

Keywords Null space control · Dynamic control · Optimization · Robotic manipulator $\cdot$ Redundancy $\cdot$ Torque minimization

\section{Introduction}

The utility of robotic manipulators is in their ability to carry out laborious tasks that involve interactions with objects, or negotiating with various external forces. This can include tasks such as lifting, pushing/pulling, bracing the manipulator against external shocks, or carrying out high-pressure blasting for cleaning surfaces. External forces on the endeffector introduce additional joint torque alongside the internal dynamic torque during task execution. Furthermore, there may be cases in which the manipulator is required to work at or above its nominal payload capacity. In such instances, careful control of the joint configuration is necessary to keep joint torques within appropriate limits.

Generally, the payload capacity of a manipulator must be balanced against other requirements such as size, weight, workspace volume, dexterity, and degrees of freedom [1]. As such, it is not always possible to increase payload capacity without sacrificing other performance measures. Therefore,

Jon Woolfrey

jonathan.woolfrey@student.uts.edu.au

1 University of Technology Sydney, 81 Broadway, Ultimo, 2007, NSW, Australia prudent control of the manipulator can reduce joint torque required for the task given these constraints. Redundant manipulators are capable of self motion, and this property can be exploited [2]. It is possible to reconfigure the manipulator such that it can transmit applied forces through the structure, and thus minimize torque on the joints.

The problem of robotic grit-blasting makes for an interesting case study of this problem. A robotic system for blasting and maintenance of the iconic Sydney Harbour Bridge had been developed at the University of Technology Sydney (UTS) [3]. A Denso VM manipulator was used originally, which had an advertised payload capacity of $13 \mathrm{~kg}$ [4]. Furthermore, the end-effector must be kept at a specific pose when operating above $11 \mathrm{~kg}$. The nozzle reaction forces during the grit-blasting process reached an estimated $106 \mathrm{~N}$ $(10.8 \mathrm{~kg})$ due to the high fluid velocities involved. Consequently, there were noted control issues during field experiments due to the "heavily laden manipulator" [5]. This large manipulator was originally chosen to cope with the high payload requirements. However, its size conflicted with the restrictions in the working environment due to the confined workspace it needed to operate within [3]. The mass of the manipulator was also raised as an issue, due to the frequency with which it had to be repositioned in the environment [6]. Some research had been conducted in anticipation of these 
large payload forces [7], but the solution did not translate easily to industrial robotic manipulators.

New research is also being conducted in to an autonomous underwater vehicle-manipulator system (AUVMS) capable of high-pressure water cleaning of submerged infrastructure $[8,9]$. Many current AUVMS have heavy manipulators mounted beneath the vehicle body to assist with passive stability. Conversely, a light-weight, top-mounted manipulator is needed for underwater cleaning robots to meet task, logistics, and stability requirements. A smaller manipulator may be feasible if the joint torques can be sufficiently managed.

Torque minimization has been thoroughly studied in previous literature, but only with respect to the internal dynamics of the system [10-17]. Applying these control methods to tasks with forces on the end-effector may lead to configurations that exacerbate the torque over the long term. Some of the earliest work on torque minimization can be found in [10]. The authors proposed a control method that utilized redundancy to locally minimize kinetic energy and avoid joint torque limits. In [11], it was shown that the minimum torque norm, weighted by the inverse of the inertia matrix, leads to a global reduction in kinetic energy in the manipulator. For future reference, this is referred to as the Minimum Kinetic Energy (MKE) method. In both cases, it was noted that dynamic level control of redundant manipulators can lead to instability in the system over long periods. This lead to the authors of [12] to devise the null space damping method. This method exponentially slows joints in the null space of the manipulator. It was also noted that the null space damping method outperforms previous methods in terms of joint torque minimization.

In [13], a method for damping joint torques in the presence of kinematic singularities was proposed. As in [12], the authors also used null space damping to ensure global stability of the system. Machine learning has also been explored for joint torque optimization [14]. Neural networks were used to generate control torques to track a given end-effector trajectory, whilst remaining bounded by the joint torque limits. More recently, quadratic programming (QP) has been used to address the minimization problem with consideration for joint, velocity, and acceleration constraints $[15,16]$. The latter used simultaneous torque and velocity minimization. This method circumvented high joint velocities in the null space that lead to instability problems as observed in [1012]. The authors in [17] developed a method of discretetime velocity control that incorporated torque optimization properties.

More recently, and perhaps more relevant, the problem of force distribution for impedance control for a mobile manipulator was proposed in [18]. Using QP, the cost function involved minimizing the input forces to the system, as well as torques in the null space. There is also methods for minimizing joint torque of industrial manipulators through trajectory planning [19]. However, this pre-planned method cannot adapt in real time as might be required by some tasks. And, once more, the authors only consider the dynamic torque without the effect of external loads.

In this study, the problem of torque minimization is considered for a redundant serial-link manipulator where large forces are applied to the end-effector of the robot. Whilst most of the literature presented focuses on a local reduction in the dynamic torque needed to drive a manipulator, this paper examines how to reduce the torque from external forces acting on the system. In addition, the method also incorporates kinetic energy minimization to reduce dynamic torque. The resultant motion is based on Weighted Least Norm (WLN) optimization, which can be used for joint limit and/or collision avoidance [20-22]. In doing so, the manipulator can simultaneously track a desired trajectory, minimize joint torque, and adhere to physical constraints. The efficacy of the proposed control method is explored in two different case studies. The first involves simulation of a heavy lifting task. Since the object is inertially coupled with the manipulator, the control method must account for the torque required to lift the object, but also the system dynamics. The second case pertains to a simulation of a high-pressure blasting process. In this scenario, the direction of forces changes based on where the nozzle points, which the manipulator must continually compensate for.

This paper is organized as follows. Section 2 covers the kinematics and dynamics of a serial link manipulator. Section 3 then covers conventional methods of torque minimization presented in previous literature. In particular, the inverse-inertia weighted torque norm control [11] is compared with the method developed here. Section 4 covers a null space control framework that will be applied to the proposed control method. In Section 5 the development of the proposed torque minimization method is presented. And in Section 4, the proposed method is shown to improve torque minimization over a conventioned MKE method for the two aforementioned scenarios.

\section{Problem Formulation}

\subsection{Serial-Link Manipulator Kinematics}

The forward kinematics of a serial link manipulator describes the position and orientation of the end-effector as a function of the joint angles:

$\mathbf{x}=\mathbf{f}(\mathbf{q})$, 
where $\mathbf{x} \in \mathbb{R}^{\mathrm{m}}$ is a vector denoting the pose of the end-effector, and $\mathbf{q} \in \mathbb{R}^{\mathrm{n}}$ is a vector of joint angles. Differentiating (1) with respect to time leads to:

$$
\begin{aligned}
\dot{\mathbf{x}} & =(\partial \mathbf{f} / \partial \mathbf{q}) \dot{\mathbf{q}} \\
& =\mathbf{J}(\mathbf{q}) \dot{\mathbf{q}} \\
\ddot{\mathbf{x}} & =\mathbf{J}(\mathbf{q}) \ddot{\mathbf{q}}+\dot{\mathbf{J}}(\mathbf{q}, \dot{\mathbf{q}}) \dot{\mathbf{q}},
\end{aligned}
$$

where $\mathbf{J}(\mathbf{q}) \in \mathbb{R}^{\mathrm{m} \times \mathrm{n}}$ is the Jacobian matrix for the manipulator. The vector $\ddot{\mathbf{x}}$ denoting the primary task is the end-effector acceleration required to track a given trajectory. If $\mathrm{m}<\mathrm{n}$, and the Jacobian has full row rank, then there are infinite joint accelerations $\ddot{\mathbf{q}}$ to achieve the required endeffector motion. Based on this, it is possible to solve an optimization problem of the form:

$$
\min _{\ddot{\mathbf{q}}}\left(\ddot{\mathbf{q}}-\ddot{\mathbf{q}}_{2}\right)^{\mathrm{T}} \mathbf{W}\left(\ddot{\mathbf{q}}-\ddot{\mathbf{q}}_{2}\right)
$$

subject to: $\ddot{\mathbf{x}}-\mathbf{J} \ddot{\mathbf{q}}-\dot{\mathbf{J}} \dot{\mathbf{q}}=\mathbf{0}$.

The solution can be derived via Lagrange multipliers, which yields the result:

$\ddot{\mathbf{q}}=\mathbf{J}_{\mathrm{W}}^{\dagger}(\ddot{\mathbf{x}}-\dot{\mathbf{J}} \dot{\mathbf{q}})+\mathbf{N}_{\mathrm{W}} \ddot{\mathbf{q}}_{2}$,

where:

- $\ddot{\mathbf{q}}_{2} \in \mathbb{R}^{\mathrm{n}}$ are joint accelerations defining a secondary, redundant task to be achieved only after $\ddot{\mathbf{x}}$ is satisfied,

- $\mathbf{W} \in \mathbb{R}^{\mathrm{n} \times \mathrm{n}}$ is a positive-definite weighting matrix,

- $\mathbf{J}_{\mathrm{W}}^{\dagger}=\mathbf{W}^{-1} \mathbf{J}^{\mathrm{T}}\left(\mathbf{J} \mathbf{W}^{-1} \mathbf{J}^{\mathrm{T}}\right)^{-1}$ is the weighted, pseudoinverse Jacobian, and

- $\quad \mathbf{N}_{\mathrm{W}}=\mathbf{I}-\mathbf{J}_{\mathrm{W}}^{\dagger} \mathbf{J}$ is the weighted null space matrix.

Note that multiplying (7) through by the Jacobian results in $\mathbf{J} \ddot{\mathbf{q}}=\ddot{\mathbf{x}}-\dot{\mathbf{J}} \dot{\mathbf{q}}$, and hence the kinematic relationship in Eq. 4 is preserved. The task defined by $\ddot{\mathbf{q}}_{2}$ will have no effect on the end-effector motion.

In some instances, $\mathbf{J}_{\mathrm{W}}^{\dagger}$ becomes ill-conditioned as the manipulator approaches a singularity. The pseudoinverse Jacobian can be modified using the well-known DampedLeast-Squares (DLS) approach [23]:

$\mathbf{J}_{\mathrm{W}, \mathrm{DLS}}^{\dagger}=\mathbf{W}^{-1} \mathbf{J}^{\mathrm{T}}\left(\mathbf{J W}^{-1} \mathbf{J}^{\mathrm{T}}+\lambda \mathbf{I}\right)^{-1}$,

where $\lambda$ is a scalar damping coefficient. This can be attenuated based on proximity to a singular configuration to mitigate task error [24].

\subsection{Manipulator Dynamics}

From a desired joint acceleration (7), the subsequent joint torques can be calculated from the manipulator dynamics:

$\boldsymbol{\tau}=\mathbf{M}(\mathbf{q}) \ddot{\mathbf{q}}+\mathbf{c}(\mathbf{q}, \dot{\mathbf{q}})+\mathbf{g}(\mathbf{q})+\boldsymbol{\tau}_{\mathrm{E}}(\mathbf{q})$ where:

- $\mathbf{M}(\mathbf{q}) \in \mathbb{R}^{\mathrm{n} \times \mathrm{n}}$ is the inertia matrix,

- $\boldsymbol{c}(\mathbf{q}, \dot{\mathbf{q}}) \in \mathbb{R}^{\mathrm{n}}$ is the vector of Coriolis and centripetal torques,

- $\mathbf{g}(\mathbf{q}) \in \mathbb{R}^{\mathrm{n}}$ is the gravity torque vector, and

- $\boldsymbol{\tau}_{\mathrm{E}}(\mathbf{q}) \in \mathbb{R}^{\mathrm{n}}$ is a vector of joint torques contributed from an external load on the end-effector.

The vector of joint torques produced from a wrench on the end-effector, $v \in \mathbb{R}^{\mathrm{m}}$, can be calculated from the manipulator Jacobian. Assuming a static configuration, and for some infinitesimally small displacements of $\Delta \mathbf{x}$ and $\Delta \mathbf{q}$, it holds that:

$\Delta \mathbf{x}=\mathbf{J} \Delta \mathbf{q}$.

Then from the principle of virtual work, the change in total energy of the system must be zero:

$\Delta \mathrm{E}=\boldsymbol{v}_{0}^{\mathrm{T}} \Delta \mathbf{x}-\boldsymbol{\tau}_{\mathrm{E}}^{\mathrm{T}} \Delta \mathbf{q}=0$,

where $\boldsymbol{\tau}_{\mathrm{E}}$ is a vector of torques needed to oppose said wrench. Substituting (10) in (11), and solving for $\boldsymbol{\tau}_{\mathrm{E}}$ :

$$
\begin{aligned}
\boldsymbol{v}_{0} \mathbf{J} \Delta \mathbf{q} & =\boldsymbol{\tau}_{\mathrm{E}}^{\mathrm{T}} \Delta \mathbf{q} \\
\boldsymbol{\tau}_{\mathrm{E}} & =\mathbf{J}^{\mathrm{T}} \boldsymbol{v}_{0} \\
& =\mathbf{J}^{\mathrm{T}} \mathbf{R}_{i} \boldsymbol{v}_{i},
\end{aligned}
$$

where $\boldsymbol{v}_{i}$ is the wrench referenced in some arbitrary frame $\{\mathrm{i}\}$, and $\mathbf{R}_{\mathbf{i}} \in \mathbb{S O}(\mathrm{m})$ is a rotation from the manipulator base frame $\{0\}$ to $\{i\}$. For $m=6$, this rotation matrix can be defined as:

$\mathbf{R}_{\mathbf{i}} \triangleq\left[\begin{array}{cc}\mathbf{R}_{0}^{\mathrm{i}}(\mathbf{q}) & \mathbf{0}_{3 \times 3} \\ \mathbf{0}_{3 \times 3} & \mathbf{R}_{0}^{\mathrm{i}}(\mathbf{q})\end{array}\right]$,

with $\mathbf{R}_{0}^{\mathrm{i}}(\mathbf{q}) \in \mathbb{S O}(3)$ being a rotation dependent on the joint configuration $\mathbf{q}$, as it exists somewhere on the kinematic structure of the manipulator.

It is evident from Eq. 14 that the joint torques needed to oppose an external loading on the manipulator is dependent on the joint state. By using redundancy in the system, it is possible to reconfigure the joints to reduce the magnitude of the torque vector $\boldsymbol{\tau}_{\mathrm{E}}$. The objective in this paper is to find a combination of joint accelerations $\ddot{\mathbf{q}}$ to achieve two outcomes. Firstly, to track a required end-effector trajectory. Secondly, to minimize both the torques required to oppose forces on the end-effector and the dynamic torque required to move the manipulator itself.

\section{Conventional Methods for Torque Minimization}

Most literature on torque minimization only considers the internal dynamics of the manipulator system. The 
introduction of large forces via $\boldsymbol{\tau}_{\mathrm{E}}$ to these control methods (in partcular, the MKE method) does not always lead to a reduction in the total joint torque over the long term. This can be seen from the simulation results in Section 6 . Furthermore, much of the early work did not account for physical constraints on the system. Some QP methods were devised that incorporated constraints on joint position, velocity, and acceleration $[15,16]$. A disdvantage to such a formulation is the lack of a closed-form solution.

\subsection{Torque Minimization in the Null Space}

In [10], a control method was proposed to locally minimize kinetic energy needed to control a redundant manipulator, as well as keep joint torques in the middle of its performance range. This resulted in the secondary redundant task $\ddot{\mathbf{q}}_{2}$ (7) as follows:

$\ddot{\mathbf{q}}_{2}=\left(\mathbf{W}^{\frac{1}{2}} \mathbf{M N}\right)^{\dagger}\left(\mathbf{W}^{\frac{1}{2}} \frac{\boldsymbol{\tau}_{\max }+\boldsymbol{\tau}_{\min }}{2}\right)$.

The diagonal elements of $\mathbf{W}$ were assigned based on torque limits for each joint. The author noted that this local kinetic energy minimization can lead to long-term instability in the system due to high joint velocities.

\subsection{Minimum Kinetic Energy (MKE) Method}

The MKE method, developed in [11], formed a foundation for later work in the area of torque minimization. This method solves a constrained optimization problem of the form:

$$
\min _{\ddot{\mathbf{q}}} 1 / 2 \boldsymbol{\tau}^{\mathrm{T}} \mathbf{M}^{-1} \boldsymbol{\tau}
$$

subject to: $\ddot{\mathbf{x}}-\mathbf{J} \ddot{\mathbf{q}}-\mathbf{J} \dot{\mathbf{q}}=\mathbf{0}$.

By substituting (9) in to (17), and solving via Lagrange multipliers, the solution is:

$\ddot{\mathbf{q}}=\mathbf{J}_{\mathrm{M}}^{\dagger}(\ddot{\mathbf{x}}-\dot{\mathbf{J}} \dot{\mathbf{q}})-\mathbf{N}_{\mathrm{M}} \mathbf{M}^{-1}\left(\mathbf{c}+\boldsymbol{\tau}_{\mathrm{E}}\right)$

This result is equivalent to Eq. 7 in which $\mathbf{W} \equiv \mathbf{M}$ and $\ddot{\mathbf{q}}_{2} \equiv \mathbf{M}^{-1}\left(\mathbf{c}+\boldsymbol{\tau}_{\mathrm{E}}\right)$. As in [11], the gravity torque $\mathbf{g}$ has been removed. This way, the manipulator configuration does not droop as it attempts to reduce the gravitational potential energy in the system. Furthermore, the original formulation did not include the term $\boldsymbol{\tau}_{\mathrm{E}}$. It was shown in [11], that Eq. 19, sans $\boldsymbol{\tau}_{\mathrm{E}}$, leads to a global minimization of kinetic energy in the manipulator system. This will be later used as a baseline to compare with the control method developed in this paper.

\subsection{Null Space Damping Method}

It has been noted that the joint control equation of the form given in Eq. 7 is inherently unstable as the null space velocities remain uncontrolled. That is, any combination of joints that do not contribute to the end-effector motion can move freely which can make the system unstable. The null space damping method was proposed in [12] to address this issue. This involved a modification to the MKE method to ensure stability. A damping term is simply appended to the redundant task like so:

$\ddot{\mathbf{q}}=\mathbf{J}_{\mathrm{M}}^{\dagger}(\ddot{\mathbf{x}}-\dot{\mathbf{J}} \dot{\mathbf{q}})-\mathbf{N}_{\mathrm{M}}\left(\mathbf{M}^{-1}\left(\mathbf{c}+\boldsymbol{\tau}_{\mathrm{E}}\right)+\beta \dot{\mathbf{q}}\right)$,

for some scalar $\beta>0$. Thus, any uncontrolled joints in the null space will slow down exponentially [12]. The authors claimed that this extension also improved torque reduction over the original MKE method.

\subsection{Simultaneous Velocity and Torque Minimization}

Due to the phenomena of high joint velocities that appear from kinetic energy minimization, the authors of [16] proposed a dual velocity and torque minimization:

$\min 1 / 2\left(\alpha \dot{\mathbf{q}}^{\mathrm{T}} \dot{\mathbf{q}}+\beta \boldsymbol{\tau}^{\mathrm{T}} \boldsymbol{\tau}\right)$.

This was solved via QP, with constraints on the joint position, velocity, and torque. Their results showed that torque minimization can be achieved whilst preventing dramatic increases in the joint velocities. Again, it ought to be noted that these four methods only consider the internal dynamics of the manipulator, and do not account for external loading.

\section{Null Space Stability and Control}

To address null space instability, the authors in [25] devised a method to control the null space velocities to a desired state. The advantage with this method is the ability to optimize a performance criterion as a function of the joint state, much like [2]. The control scheme is briefly presented here, and in Section 5 the framework is applied to simultaneously minimize torque for trajectory tracking whilst incorporating physical system constraints. First, the control accelerations can be defined as:

$\ddot{\mathbf{q}}=\mathbf{J}^{\dagger}(\ddot{\mathbf{x}}-\dot{\mathbf{J}} \dot{\mathbf{q}})+\ddot{\mathbf{q}}_{\mathrm{N}}$,

where $\ddot{\mathbf{q}}_{\mathrm{N}} \in \mathbb{R}^{\mathrm{n}}$ is a redundant task such that $\mathbf{J} \ddot{\mathbf{q}}_{\mathrm{N}}=\mathbf{0}$, and $\mathbf{J}^{\dagger}$ is the unweighted Moore-Penrose pseudoinverse Jacobian. To control the null space velocities, the following was proposed [25]:

$\ddot{\mathbf{q}}_{\mathrm{N}}=\mathbf{N}\left(\ddot{\mathbf{q}}_{\mathrm{d}}+\mathbf{K}_{\mathrm{N}} \mathbf{e}_{\mathrm{N}}\right)-\left(\dot{\mathbf{J}}^{\dagger}+\mathbf{J}^{\dagger} \dot{\mathbf{J}} \mathbf{J}^{\dagger}\right) \mathbf{J}\left(\dot{\mathbf{q}}_{\mathrm{d}}-\dot{\mathbf{q}}\right)$, 
where:

- $\quad \dot{\mathbf{q}}_{\mathrm{d}}$ is the desired joint velocity,

- $\mathbf{N}$ is the unweighted null space matrix, that is $\mathbf{N} \equiv \mathbf{N}_{\mathrm{W}}$ for $\mathbf{W}=\mathbf{I}$,

- $\mathbf{K}_{\mathrm{N}}$ is a positive-definite gain matrix, and

- $\quad \mathbf{e}_{\mathrm{N}}=\mathbf{N}\left(\dot{\mathbf{q}}_{\mathrm{d}}-\mathbf{q}\right)$ is the joint velocity error projected on to the null space.

By taking the time derivative of $\mathbf{e}_{\mathrm{N}}$, and substituting in Eqs. 22 and 23:

$$
\begin{aligned}
\dot{\mathbf{e}}_{\mathrm{N}} & =\mathbf{N}\left(\ddot{\mathbf{q}}_{\mathrm{d}}-\ddot{\mathbf{q}}\right)-\mathbf{J}^{\dagger} \dot{\mathbf{J}} \mathbf{e}_{\mathrm{N}}-\left(\dot{\mathbf{J}}^{\dagger}+\mathbf{J}^{\dagger} \dot{\mathbf{J} J} \mathbf{J}^{\dagger}\right) \mathbf{J}\left(\dot{\mathbf{q}}_{\mathrm{d}}-\dot{\mathbf{q}}\right) \\
& =\mathbf{N}\left(\ddot{\mathbf{q}}_{\mathrm{d}}-\ddot{\mathbf{q}}_{\mathrm{N}}\right)-\mathbf{J}^{\dagger} \mathbf{J} \mathbf{e}_{\mathrm{N}}-\left(\dot{\mathbf{J}}^{\dagger}+\mathbf{J}^{\dagger} \dot{\mathbf{J}} \mathbf{J}^{\dagger}\right) \mathbf{J}\left(\dot{\mathbf{q}}_{\mathrm{d}}-\dot{\mathbf{q}}\right) \\
& =-\mathbf{N} \mathbf{K}_{\mathrm{N}} \mathbf{e}_{\mathrm{N}}-\mathbf{J}^{\dagger} \dot{\mathbf{J}} \mathbf{e}_{\mathrm{N}} .
\end{aligned}
$$

A Lyapunov candidate function can be defined as the sumof-squared errors:

$\mathrm{V}=1 / 2 \mathbf{e}_{\mathrm{N}}^{\mathrm{T}} \mathbf{e}_{\mathrm{N}}$,

then the time-derivative is:

$$
\begin{aligned}
\dot{\mathrm{V}} & =\mathbf{e}_{\mathrm{N}}^{\mathrm{T}} \dot{\mathbf{e}}_{\mathrm{N}} \\
& =-\mathbf{e}_{\mathrm{N}}^{\mathrm{T}} \mathbf{N K}_{\mathrm{N}} \mathbf{e}_{\mathrm{N}}-\mathbf{e}_{\mathrm{N}}^{\mathrm{T}} \mathbf{J}^{\dagger} \dot{\mathbf{J}} \mathbf{e}_{\mathrm{N}} \\
& =-\mathbf{e}_{\mathrm{N}}^{\mathrm{T}} \mathbf{K}_{\mathrm{N}} \mathbf{e}_{\mathrm{N}},
\end{aligned}
$$

since $\mathbf{N}$ is symmetric and idempotent, and $\mathbf{J N}=\mathbf{N} \mathbf{J}^{\dagger}=\mathbf{0}$. Equation 28 is negative if $\mathbf{K}_{\mathrm{N}}$ is positive-definite, and hence $\dot{\mathbf{q}}$ converges to $\dot{\mathbf{q}}_{\mathrm{d}}$ in the space of $\mathbf{N}$.

\section{Control Method for Torque Minimization with External Loading}

In this section, a new control method is proposed to minimize the joint torque with forces on the manipulator's end-effector. This method builds on the null space velocity control in Section 4. This ensures stability, and circumvents the fictitious damping forces in the null space damping method that interferes with the redundant task. Rather than locally minimizing the total torque, as per the MKE method, the objective function is defined as a weighted sum. The first component is to minimize the norm of torques needed to oppose the forces on the end-effector. The second component minimizes dynamic torque. In this manner, the manipulator will align itself against the external force, but also minimize the effort required to achieve this.

\subsection{Derivation of Desired Velocity, Acceleration}

To stabilize the null space velocities, a desired joint velocity must be defined. In [25], the desired velocity was equivalent to the task for maximimizing manipulability. Here, the desired velocity is constructed via the WLN solution to the differential kinematics (7). This way, both the main task and redundant task can be made to conform to physical constraints. The desired joint velocity and its time-derivative can be expressed generally as:

$\dot{\mathbf{q}}_{\mathrm{d}}=\mathbf{J}_{\mathrm{W}}^{\dagger} \dot{\mathbf{x}}+\mathbf{N}_{\mathrm{W}} \dot{\mathbf{y}}$

$\ddot{\mathbf{q}}_{\mathrm{d}}=\mathbf{J}_{\mathrm{W}}^{\dagger}(\ddot{\mathbf{x}}-\mathbf{J} \dot{\mathbf{y}})+\dot{\mathbf{J}}_{\mathrm{W}}^{\dagger}(\dot{\mathbf{x}}-\mathbf{J} \dot{\mathbf{y}})+\mathbf{N}_{\mathrm{W}} \ddot{\mathbf{y}}$,

where the secondary task $\dot{\mathbf{y}}, \ddot{\mathbf{y}} \in \mathbb{R}^{\mathrm{n}}$ is formulated as a sum of two components that will be used to minimize the static and dynamic torque components:

$\dot{\mathbf{y}}=\alpha_{1} \dot{\mathbf{y}}_{1}+\alpha_{2} \dot{\mathbf{y}}_{2}$,

in which $\alpha_{1}$ and $\alpha_{2}$ are scalars to weight the two vectors. Also, multiplying (30) through by the Jacobian yields $\mathbf{J} \ddot{\mathbf{q}}_{\mathrm{d}}=$ $\ddot{\mathbf{x}}-\dot{\mathbf{J}} \dot{\mathbf{q}}_{\mathrm{d}}$ thus maintaining the kinematic relationship denoted by Eq. 4. Note that it is possible to define $\dot{\mathbf{q}}_{\mathrm{d}}=\dot{\mathbf{y}}$, but by Eq. 29 the joints can be made to comply with physical constraints through $\mathbf{J}_{\mathrm{W}}^{\dagger}$.

By taking $\dot{\mathbf{y}}_{1}$ as proportional to the gradient vector of some scalar cost function $\mathrm{h}(\mathbf{q})$, the application of Eq. 29 will optimize this in the null space of the manipulator:

$\dot{\mathbf{y}}_{1}=\nabla \mathrm{h}(\mathbf{q})$.

Choosing $\alpha_{1}<0$ in Eq. 31 will minimize $\mathrm{h}(\mathbf{q})$. This is otherwise known as the Gradient Projection Method (GPM) [2]. Its time derivative is then:

$$
\begin{aligned}
\ddot{\mathbf{y}}_{1} & =\frac{\mathrm{d}}{\mathrm{dt}}(\nabla \mathrm{h}(\mathbf{q})) \\
& =\frac{\mathrm{d}}{\mathrm{dq}}(\nabla \mathrm{h}(\mathbf{q})) \dot{\mathbf{q}} \\
& =\mathbf{H}(\mathbf{q}) \dot{\mathbf{q}},
\end{aligned}
$$

where $\mathbf{H}(\mathbf{q})$ is the Hessian matrix of $h(\mathbf{q})$.

To minimize the joint torques caused by a wrench $\boldsymbol{v}_{\mathrm{i}}$ on the manipulator, consider the following cost function:

$\mathrm{h}(\mathbf{q})=1 / 2 \hat{\boldsymbol{\tau}}_{\mathrm{E}}^{\mathrm{T}} \mathbf{G} \hat{\boldsymbol{\tau}}_{\mathrm{E}}$

where:

$-\quad \hat{\boldsymbol{\tau}}_{\mathrm{E}}=\mathbf{J}^{\mathrm{T}} \mathbf{R}_{\mathrm{i}} \hat{v}_{\mathrm{i}}$

- $\hat{v}_{\mathrm{i}}=\boldsymbol{v}_{\mathrm{i}} /\left\|\boldsymbol{v}_{\mathrm{i}}\right\|$ is the unit vector of $\boldsymbol{v}_{\mathrm{i}}$ which defines its direction, and

- $\mathbf{G} \in \mathbb{R}^{\mathrm{n} \times \mathrm{n}}$ is a positive-definite weighting matrix used to give priority to joints with higher torque capacity, or better mechanical advantage.

The gradient of Eq. 36 with respect to $\mathbf{q}$ is:

$$
\begin{aligned}
\nabla \mathrm{h}(\mathbf{q}) & =\left(\partial \hat{\boldsymbol{\tau}}_{\mathrm{E}} / \partial \mathbf{q}\right)^{\mathrm{T}} \mathbf{G} \hat{\boldsymbol{\tau}}_{\mathrm{E}} \\
\frac{\partial \mathrm{h}}{\partial \mathrm{q}_{\mathrm{j}}} & =\hat{v}_{\mathrm{i}}^{\mathrm{T}}\left(\frac{\partial \mathbf{R}_{\mathrm{i}}^{\mathrm{T}}}{\partial \mathrm{q}_{\mathrm{j}}} \mathbf{J}+\mathbf{R}_{\mathrm{i}}^{\mathrm{T}} \frac{\partial \mathbf{J}}{\partial \mathrm{q}_{\mathrm{j}}}\right) \mathbf{G} \mathbf{J}^{\mathrm{T}} \mathbf{R}_{\mathrm{i}} \hat{v}_{\mathrm{i}}, \quad \mathrm{j} \in\{1, \ldots, \mathrm{n}\} .
\end{aligned}
$$


The Hessian for Eq. 36 is difficult to calculate, so for practical purposes it is sufficient to use backwards differencing to approximate the time-derivative:

$\ddot{\mathbf{y}}_{1}(\mathrm{t}) \approx \Delta \mathrm{t}^{-1}\left(\dot{\mathbf{y}}_{1}(\mathrm{t})-\dot{\mathbf{y}}_{1}(\mathrm{t}-\Delta \mathrm{t})\right)$,

where $\Delta \mathrm{t}^{-1}$ is the control frequency.

The joint motion in the redundant portion of the manipulator from $\dot{\mathbf{y}}_{1}, \ddot{\mathbf{y}}_{1}$ may locally increase the dynamic torque. Therefore, the second portion of the redundant task can be used to reduce the local accelerations caused by the torque components $\mathbf{c}$ and $\boldsymbol{\tau}_{\mathrm{E}}$ :

$\ddot{\mathbf{y}}_{2}=-\mathbf{M}^{-1}\left(\mathbf{c}+\boldsymbol{\tau}_{\mathrm{E}}\right)$.

To find the velocity level term for this second vector, discrete-time integration can be used:

$\dot{\mathbf{y}}_{2}(\mathrm{t}) \approx \dot{\mathbf{y}}_{2}(\mathrm{t}-\Delta \mathrm{t})+\Delta \mathrm{t} \ddot{\mathbf{y}}_{2}(\mathrm{t}-\Delta \mathrm{t})$

This second vector component $\ddot{\mathbf{y}}_{2}$ is similar to the MKE method (19). Equation 31 is thus a weighted sum between minimizing the norm of $\boldsymbol{\tau}_{\mathrm{E}}$ and reducing the local dynamic torque. The scalars $\alpha_{1}$ and $\alpha_{2}$ can be chosen based on the relative magnitude of the corresponding torques.

\subsection{Incorporation of Physical Constraints}

The control equations (7), (29), and (30) represent a weighted-least-norm solution for the joint motion. It is possible to account for physical constraints in the solution via the construction of the weighting matrix $\mathbf{W}$. Some examples include joint limit avoidance [20], self-collision avoidance [21], and obstacle avoidance [22]. In this paper, W is constructed for joint limit avoidance as per [20]. The weighting matrix is modified using the following cost function:

$\mathrm{d}\left(\mathrm{q}_{\mathrm{i}}\right)=\frac{\left(\mathrm{q}_{\mathrm{i}, \max }-\mathrm{q}_{\mathrm{i}, \text { min }}\right)\left(2 \mathrm{q}_{\mathrm{i}}-\mathrm{q}_{\mathrm{i}, \text { max }}-\mathrm{q}_{\mathrm{i}, \text { min }}\right)}{\gamma_{\mathrm{i}}\left(\mathrm{q}_{\mathrm{i}, \max }-\mathrm{q}_{\mathrm{i}}\right)^{2}\left(\mathrm{q}_{\mathrm{i}}-\mathrm{q}_{\mathrm{i}, \text { min }}\right)^{2}}$,

in which magnitude of this function grows to infinity near joint limits, causing joints to slow down in their approach. In this paper, the joint limit cost function is combined with the inertia matrix $\mathbf{M}$ :

$$
\begin{aligned}
& \mathbf{W}_{\mathrm{ij}}=\mathbf{M}_{\mathrm{ij}} \quad \forall \mathrm{i} \neq \mathrm{j} \\
& \mathbf{W}_{\mathrm{ii}}=\left\{\begin{array}{cl}
\mathbf{M}_{\mathrm{ii}}+\left|\mathrm{d}\left(\mathrm{q}_{\mathrm{i}}\right)\right| & \text { for } \Delta \mathrm{d}\left(\mathrm{q}_{\mathrm{i}}\right)>0 \\
\mathbf{M}_{\mathrm{ii}} & \text { otherwise }
\end{array}\right.
\end{aligned}
$$

The inertia component of this weighting matrix will help minimize the kinetic energy in the manipulator. Furthermore, the element $\mathbf{W}_{\mathrm{ii}}$ will approach infinity as a joint moves toward its limit. Thus through Eqs. 29 and 30, joints moving away from their limits will be given preference in the solution.

\section{Case Studies}

The proposed control method is examined through two different case studies. The first involves a manipulator lifting and moving a heavy object. Here, the manipulator must overcome gravitational forces when raising the object, but must also maintain a controlled descent when lowering it (Fig. 1). Additionally, the object is inertially coupled with the manipulator and dynamic forces must be accounted for while moving. The second scenario involves robotic high-pressure blasting where the nozzle reaction forces continually push back against the manipulator. Furthermore, the direction of these forces changes depending on where the nozzle points as required by the task (Fig. 5).

\subsection{Case Study 1: Torque Minimization for Heavy Lifting}

Outline In this first case study, a dynamic model of the Sawyer robot by Rethink Robotics with 7DOF is simulated.

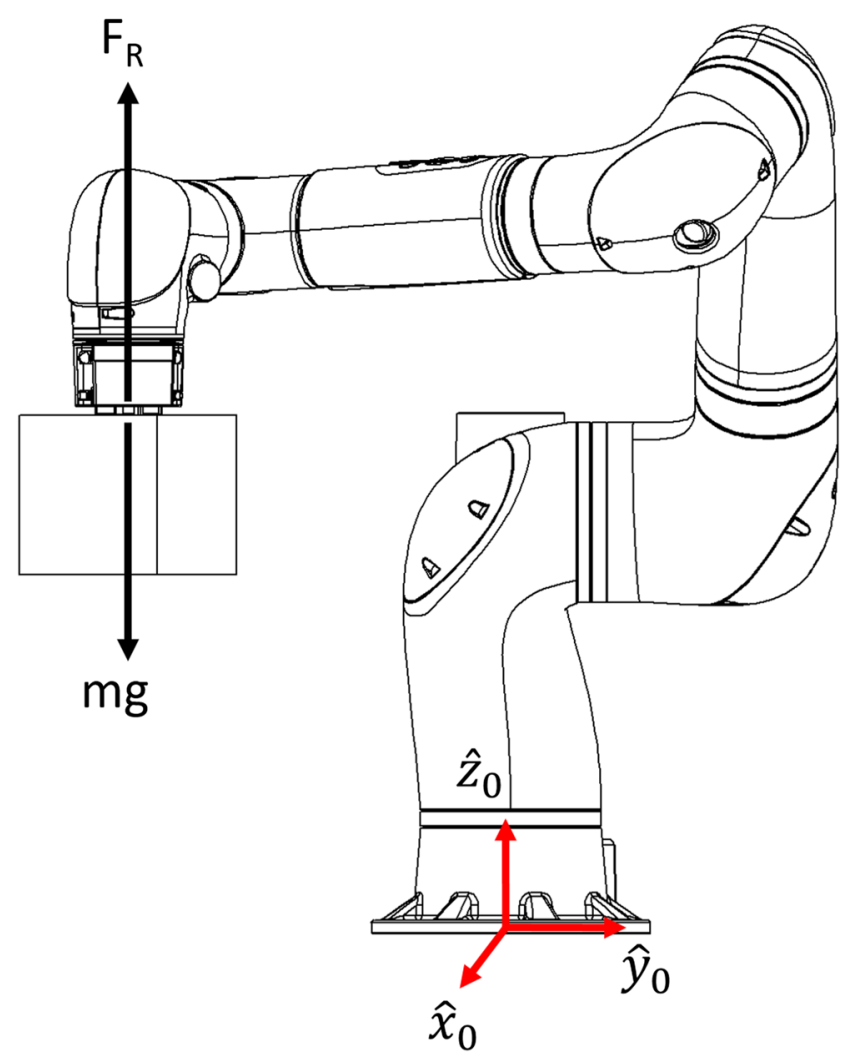

Fig. 1 When lifting, the manipulator must generate a force opposite the payload's gravitational force 
Table 1 Physical properties of the 7DOF manipulator used for the heavy-lifting simulation

\begin{tabular}{llllllll}
\hline & Joint & & & & & & \\
& 1 & 2 & 3 & 4 & 5 & 6 & 7 \\
\hline Mass (kg) & 5.3 & 4.5 & 1.7 & 2.5 & 1.1 & 1.6 & $\begin{array}{l}0.3 \\
\text { Max. Torque (N) }\end{array}$ \\
\hline
\end{tabular}

The task requirement here is to lift a heavy object to a height of $650 \mathrm{~mm}$, move it $700 \mathrm{~mm}$ laterally, then lower it back down. The wrench on the end-effector is then denoted with respect to the base frame $\{0\}$ :

$\boldsymbol{v}_{0}=\left[\begin{array}{llllll}0 & 0 & F_{\mathrm{R}} & 0 & 0 & 0\end{array}\right]^{\mathrm{T}}$.

As such, $\mathbf{R}=\mathbf{I}(12)$ and $\partial \mathbf{R} / \partial \mathrm{q}_{j}=\mathbf{0}$ (30). The joint torques generated by the weight of the object on the joints is then:

$\boldsymbol{\tau}_{\mathrm{E}}=\mathbf{J}^{\mathrm{T}} \boldsymbol{v}_{0}$.

The object being lifted is also inertially coupled with the manipulator itself. This will introduce additional dynamic torques in the manipulator that must be balanced against the torque minimization from the external loading. Table 1 gives some of the physical properties of the manipulator used in the case study. Table 2 gives the parameters used in the control equations. The weight of the object is taken as $\mathrm{F}_{\mathrm{R}}=60 \mathrm{~N}(6 \mathrm{~kg})$ which is 1.5 times greater than the nominal payload capacity [26].

\subsubsection{Results}

Figure 2 shows a plot of the sum of the absolute joint torques for the lifting scenario. The method of torque minimization proposed in this paper reduces the total torque required for the task compared to the MKE method. Figure 3. shows screenshots of an animation for the lifting task. From 1s to $3 \mathrm{~s}$, the manipulator brings joint 4 up over itself to reduce torque. A distinct drop in torque for joint 2 can be seen from approximately $5 \mathrm{~s}$ to 10 s (Fig. 4a). A careful examination of Fig. 3 reveals why. The axis of joint 2 operates on a plane that is orthogonal to the force vector from the payload, which can cause high torques. The proposed control method redirects the axis of joint 2 in order to reduce the distance between it and the force vector on the end-effector, thus reducing the resultant torque. Since the arm has large tolerance on the joint torque and joint angles, neither control method exceeded limits.

\subsection{Case Study 2: Torque Minimization for Robotic High-Pressure Blasting}

\subsubsection{Outline}

In the second case study, a 6DOF manipulator is considered for high-pressure blasting of a flat surface. By exploiting some of the task properties, the manipulator can be tricked in to performing as a 9DOF system. A nozzle is mounted on the 6th link, in which the nozzle outlet is coincident with frame $\{6\}$ in the kinematic model (Fig. 5a). The manipulator is required to direct the fluid stream at a location on a surface with a precise offset distance for optimal cleaning. Therefore, it is convenient to denote a virtual end-effector at the optimal cleaning distance offset from $\{6\}$. Also, some leniency is tolerable in the orientation of this virtual endeffector. Thus, a virtual ball joint can be appended to the model, giving an additional 3DOF for a total of 9DOF. These will have no effect on the direction of reaction forces. This also has the added advantage that the angle of attack of the working fluid with respect to the surface may be controlled (Fig. 5).

It should be noted that joints $\mathrm{q}_{7}, \mathrm{q}_{8}$ and $\mathrm{q}_{9}$ are virtual and hence have no mass. As such, $\operatorname{rank}(\mathbf{M})=6$ and the inertia matrix cannot be inverted. It is possible to add miniscule values to the diagonal elements $\mathbf{M}_{\mathrm{ii}}$ for $\mathrm{i} \in\{7,8,9\}$ to enable inversion of $\mathbf{M}$ without any noticeable effects on system performance. However, if one of the virtual joints is near its limit, then the weighting matrix as defined in Eqs. 13 and 44 becomes ill-conditioned. Since the mass of this manipulator is small (Table 3), for this scenario it is sufficient to define the weighting matrix $\mathbf{W}$ as:

$\mathbf{W}_{\mathrm{ii}}=\left\{\begin{array}{cl}1+\left|\mathrm{d}\left(\mathrm{q}_{\mathrm{i}}\right)\right| & \text { for } \Delta \mathrm{d}\left(\mathrm{q}_{\mathrm{i}}\right)>0 \\ 1 & \text { otherwise. }\end{array}\right.$

To oppose the nozzle reaction forces $F_{R}$, the manipulator must generate a force along the positive $\mathrm{z}$-axis of frame $\{6\}$ (Fig. 5). Hence the wrench with respect to $\{6\}$ is given as:

$\boldsymbol{v}_{6}=\left[\begin{array}{llllll}0 & 0 & F_{\mathrm{R}} & 0 & 0 & 0\end{array}\right]^{\mathrm{T}}$.

Then, from (11), the torque required to oppose the nozzle reaction forces is:

$$
\begin{aligned}
\boldsymbol{\tau}_{\mathrm{E}} & =\mathbf{J}^{\mathrm{T}} \mathbf{R}_{6} \boldsymbol{v}_{6} \\
\mathbf{R}_{6} & =\left[\begin{array}{cc}
\mathbf{R}_{0}^{6} & \mathbf{0}_{3 \times 3} \\
\mathbf{0}_{3 \times 3} & \mathbf{R}_{0}^{6}
\end{array}\right] .
\end{aligned}
$$

Table 2 Parameters used in the simulation of heavy-lifting

\begin{tabular}{llllllll}
\hline & Parameter & & & & & \\
& $\mathrm{F}_{\mathrm{R}}$ & Frame & $\mathbf{G}$ & $\alpha_{1}$ & $\alpha_{2}$ & $\mathbf{K}_{\mathrm{N}}$ & $\beta$ \\
\hline Value & $60 \mathrm{~N}$ & $\{0\}$ & $\mathbf{I}$ & -10 & 0.05 & 4 & 1 \\
Equation & $(15)$ & $(14)$ & $(36)$ & $(31)$ & $(31)$ & $(23)$ & $(20)$ \\
\hline
\end{tabular}


Fig. 2 Sum of absolute joint torques for the heavy-lifting case study. The proposed control method reduces the overall torque required for the task

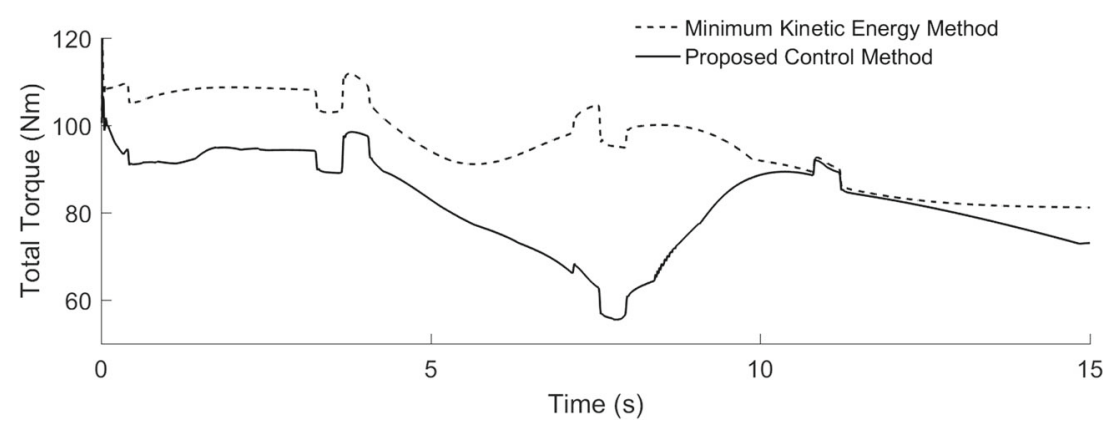

Since the payload capacity of the manipulator is much smaller than that used in [3-7], the nozzle reaction forces here are taken as $F_{R}=30 N(3 \mathrm{~kg})$. This is 6 times greater than the advertised payload capacity of the igus Robolink WR of $0.5 \mathrm{~kg}$ [27], assuming the mechanical strength is greater than the joint torque limits.

For the task, the manipulator is required to trace a rectangle within its workspace $300 \mathrm{~mm}$ wide and $150 \mathrm{~mm}$ tall. In doing so, the initial and final pose of the endeffector are the same. It is expected, then, that the torque minimization method proposed in this paper will autonomously reconfigure the joints to reduce the overall torque once it returns to its starting position. As a baseline, the MKE method with null space damping (20) is used to compare outcomes. Due to the low inertia of the manipulator $\left\|\boldsymbol{\tau}_{\mathrm{E}}\right\| \gg\|\mathbf{M} \ddot{\mathbf{q}}+\mathbf{c}\|$ and the scalar $\alpha_{2}=0$ (31). The control parameters were manually adjusted to achieve desirable outcomes (Table 4).

\subsubsection{Results}

Screenshots for the blasting simulation can be seen in Fig. 6 . The torque minimization method proposed in this paper reduces the overall torque from an external load on the endeffector (Fig. 7). In contrast, the MKE method sometimes leads to configurations that amplify the torques $\boldsymbol{\tau}_{\mathrm{E}}$ from this external loading. Furthermore, the MKE method appears to have different results depending on initial conditions. Figure 7 shows a plot of the sum of absolute joint torques for 2 different initial joint configurations. In one case, the torque from the final configuration is larger than its starting conditions for the same end-effector pose.

The proposed control method, however, results in an overall reduction of torque for both initial states. In both situations, the joint torques converge to a similar result. Furthermore, in Fig. 8a it can be seen that the joint torques are sufficiently reduced to remain within torque limits

Minimum Kinetic Energy Method:
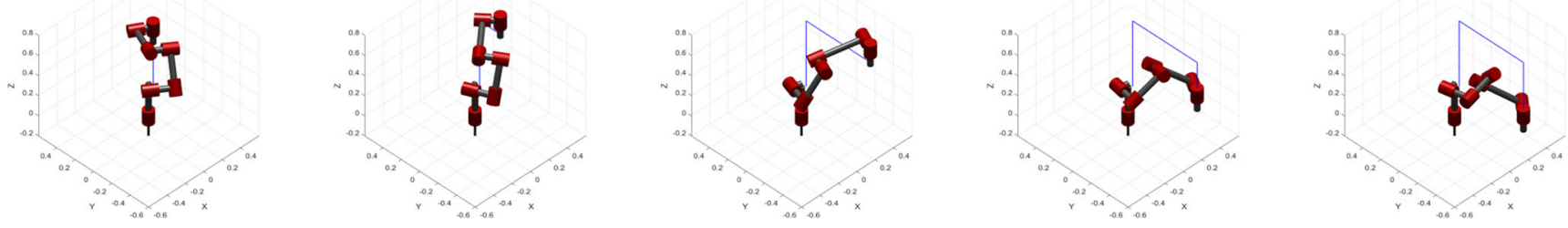

Proposed Control Method:
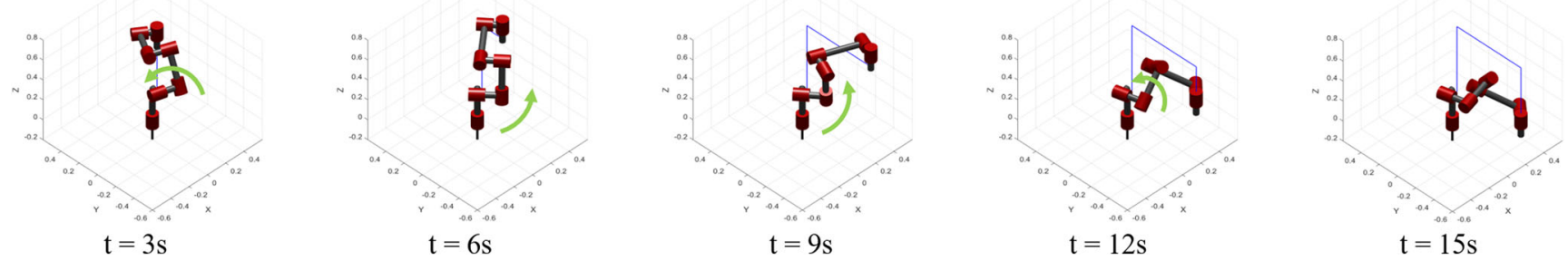

Fig. 3 Screenshots from the heavy-lifting simulation. Using the proposed control method (right), the manipulator brings joint 4 up over the payload to assist in lifting at $t=3 \mathrm{~s}$. From $t=6 \mathrm{~s}$ to $t=9 \mathrm{~s}$, the axis

of joint 2 is redirected toward the force vector of the payload, resulting in a reduction in torque (see also Fig. 4a) 
Fig. 4 Joint torques and angles for the heavy-lifting simulation. The proposed control method rearranges the joints to reduce joint torque, and assist with lifting a heavy object
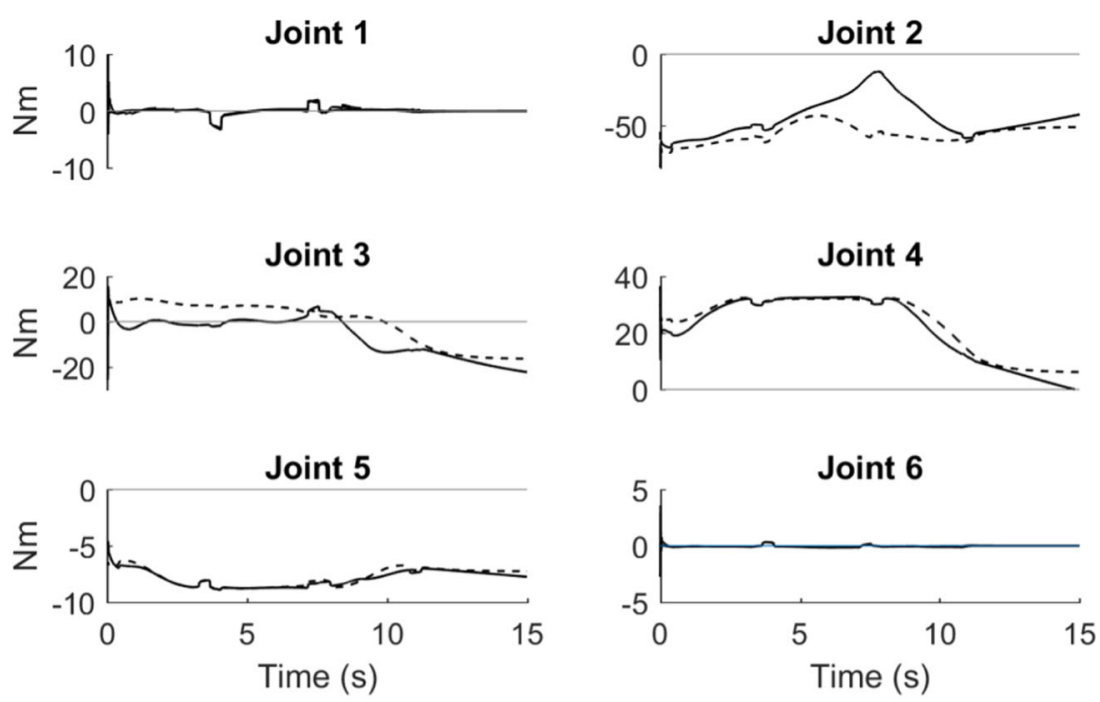

(a) Plot of individual joint torques.
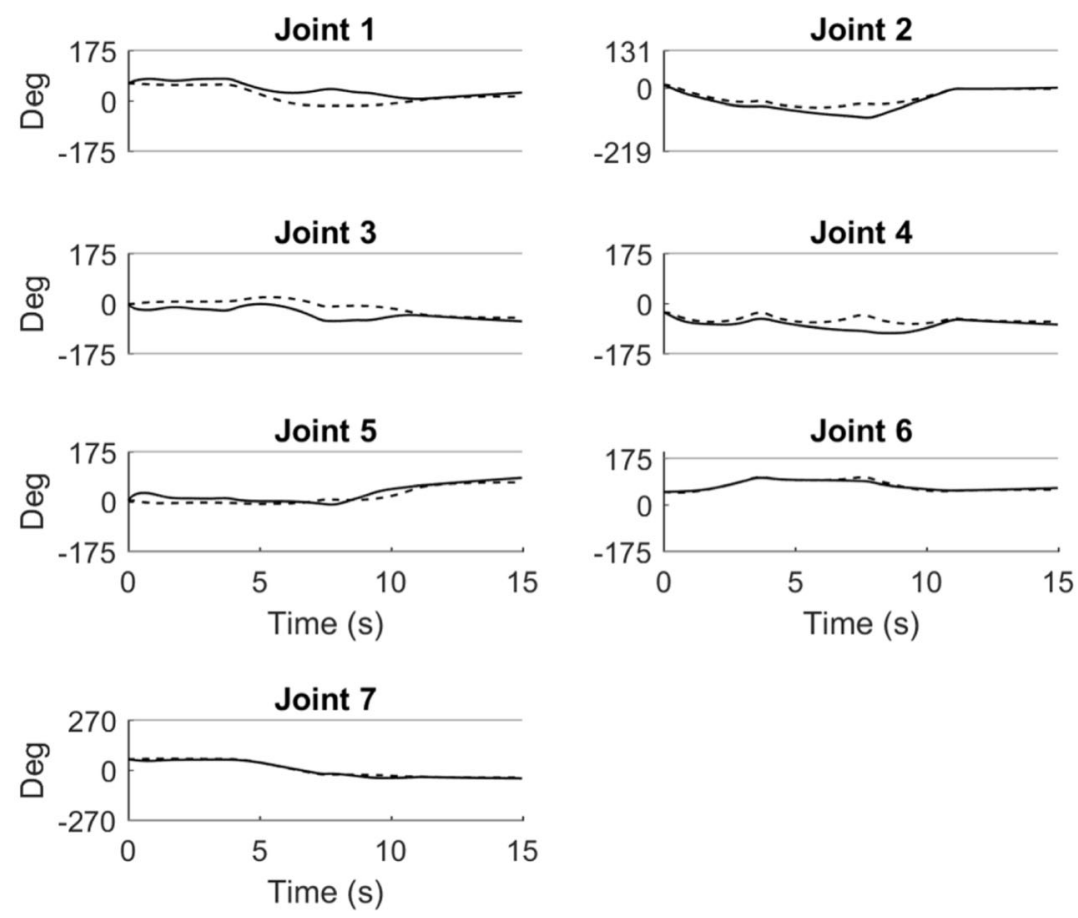

(b) Plot of individual joint angles.

(Table 3). Joint torques remained feasible even when the force on the end-effector was 6 times the nominal payload capacity. Figure $8 \mathrm{~b}$ compares joint angles using the MKE method and the proposed control method. The joint limits are violated using the MKE method for joints 5, 6 and 8. The method developed in this paper can simultaneously adhere to joint limits and minimize the overall torque as intended.

\section{Discussion}

\subsection{Considerations and Limitations}

Part of the redundant task (31) involves minimizing the norm of the torque vector $\boldsymbol{\tau}_{\mathrm{E}}$ that results from a wrench $\boldsymbol{v}$ on the end-effector (14). However, there may be situations in 
Fig. 5 When blasting, the manipulator must oppose reaction forces in frame $\{6\}$ of the robot. The blasting point can be modelled as a virtual ball joint to control the angle-of-attack, giving an additional 3DOF to the system

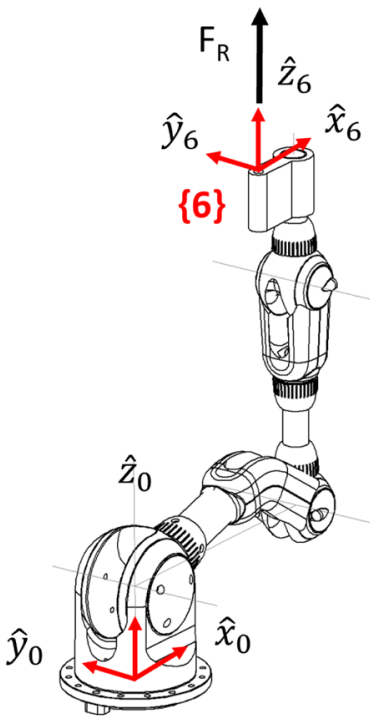

$\{0\}$

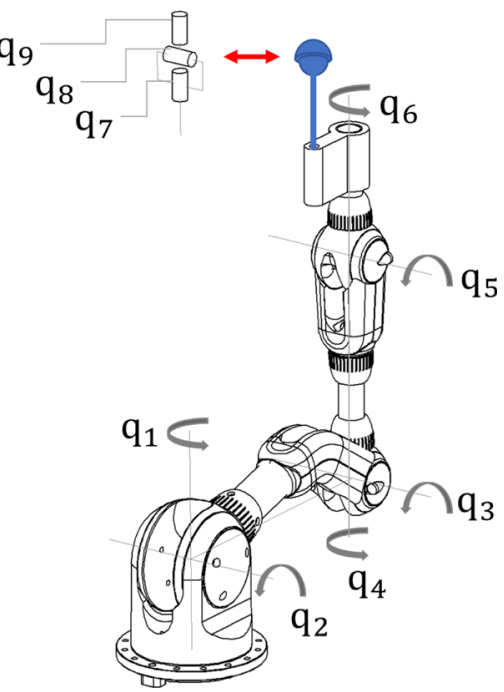

(a) Reference frames on the robot.

(b) Real and virtual joints.

which we may want to utilize this force to assist in motion. The inclusion of the term $-\alpha_{2} \mathbf{M}^{-1} \boldsymbol{\tau}_{\mathrm{E}}$ (31) performs this function to a degree, however (37) is working to minimize $\boldsymbol{\tau}_{\mathrm{E}}$. Consider the blasting scenario where we wish to clean a curved surface. We may want to stiffen the manipulator when moving forward in the direction of the nozzle to reduce torque, but relax the manipulator when moving back to take advantage of the nozzle reaction forces.

Furthermore, the redundant task is defined by the gradient to the cost function (36). This will cause the joint state of the manipulator to converge to a local minimum of this function. The joint state here may not be adequate given the task requirements, even though a better local or global minimum may exist. Therefore, the secondary task may not be able to achieve a joint configuration that sufficiently minimizes the joint torque for the task and payload required. Nevertheless, the simulations showed good results, particularly with the blasting scenario in which the manipulator was constrained by relatively narrow joint limits and large end-effector forces.

Lastly, there is also the matter of choosing adequate control parameters for $\alpha_{1}, \alpha_{2}$, and $\mathbf{K}_{\mathrm{N}}$. In this paper, these values were manually adjusted to achieve the desired results given the task requirements. For $\alpha_{1}=0$, this is effectively

Minimum Kinetic Energy Method:
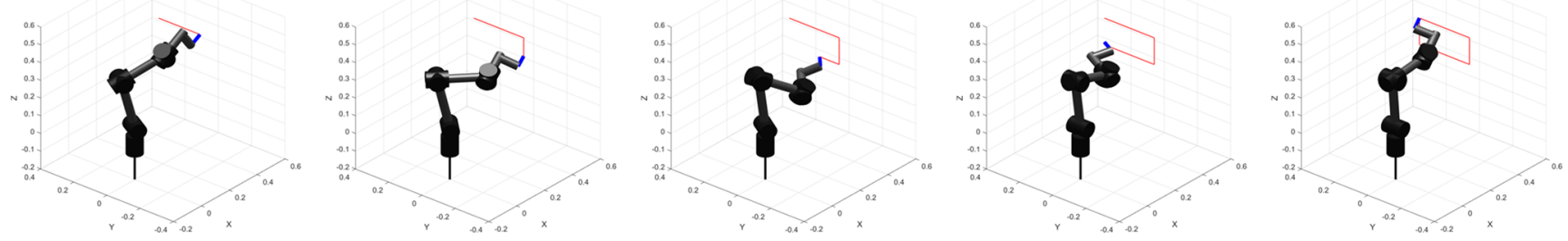

Proposed Control Method:
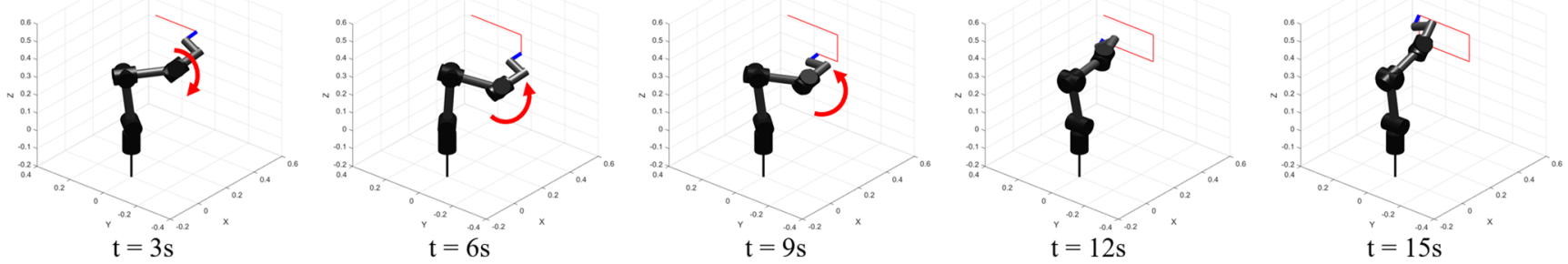

Fig. 6 Screenshots from the robotic blasting simulation. Using the proposed control method, the manipulator reconfigures itself to brace against the nozzle reaction force 
Table 3 Physical properties of the 6DOF manipulator used for the blasting simulation

\begin{tabular}{lllllll}
\hline & Joint & & & & & \\
\cline { 2 - 7 } & 1 & 2 & 3 & 4 & 5 & 6 \\
\hline Mass (kg) & 0.57 & 0.87 & 0.12 & 0.27 & 0.12 & 0.35 \\
Max. Torque (N) & 10 & 20 & 12 & 5 & 12 & 5 \\
\hline
\end{tabular}

the MKE method. Increasing the magnitude of $\alpha_{1}$ increases the speed with which the arm optimizes its configuration. This will decrease the effects of the static torque $\boldsymbol{\tau}_{\mathrm{E}}$, but increase the kinetic energy in the system which $\ddot{\mathbf{y}}_{2}$ is trying to reduce. The interaction between components $\ddot{\mathbf{y}}_{1}$ and $\ddot{\mathbf{y}}_{2}$ and how best to balance them via their respective weights $\alpha_{1}$ and $\alpha_{2}$ is not yet fully understood, and may be a point of future study.

\subsection{Mathematical Relationship to Cartesian Stiffness Control}

Cartesian stiffness control is a prominent method for minimizing the deflection of the end-effector when subject to external forces [28-32]. Given a desired end-effector stiffness, often specified with respect to the base frame, a subsequent joint configuration can be optimized [30-32]. This inverse kinematics approach implies that the either the manipulator is static, or the motion is pre-planned in the joint space.

Conversely, in this paper a torque minimization approach was proposed for real-time control of a manipulator, capable of trajectory tracking. However, there exists some mathematical relationships between this and Cartesian stiffness control that may be an avenue of future investigation.

In Cartesian stiffness control, the convention is to define a Cartesian stiffness matrix $\mathbf{K}_{\mathrm{c}}$, which maps a deflection of the end-effector $\Delta \mathbf{x}$ to a wrench $\boldsymbol{v}$ :

$\Delta \boldsymbol{v}=\mathbf{K}_{\mathrm{c}} \Delta \mathbf{x}$

Similarly, the deflection of the joints $\Delta \mathbf{q}$ is mapped to the (static) joint torques $\boldsymbol{\tau}_{\mathrm{E}}$ by the joint stiffness matrix $\mathbf{K}_{\mathrm{q}}$ :

$\Delta \boldsymbol{\tau}_{\mathrm{E}}=\mathbf{K}_{\mathrm{q}} \Delta \mathbf{q}$.

Table 4 Parameters used in the simulation of robotic blasting

\begin{tabular}{llllllll}
\hline & \multicolumn{1}{l}{ Parameter } \\
\cline { 2 - 8 } & $\mathrm{F}_{\mathrm{R}}$ & Frame & $\mathbf{G}$ & $\alpha_{1}$ & $\alpha_{2}$ & $\mathbf{K}_{\mathrm{N}}$ & $\beta$ \\
\hline Value & $30 \mathrm{~N}$ & $\{6\}$ & $\mathbf{I}$ & -4 & 0 & 5 & 2 \\
Equation & $(48)$ & $(14)$ & $(36)$ & $(31)$ & $(31)$ & $(23)$ & $(20)$ \\
\hline
\end{tabular}

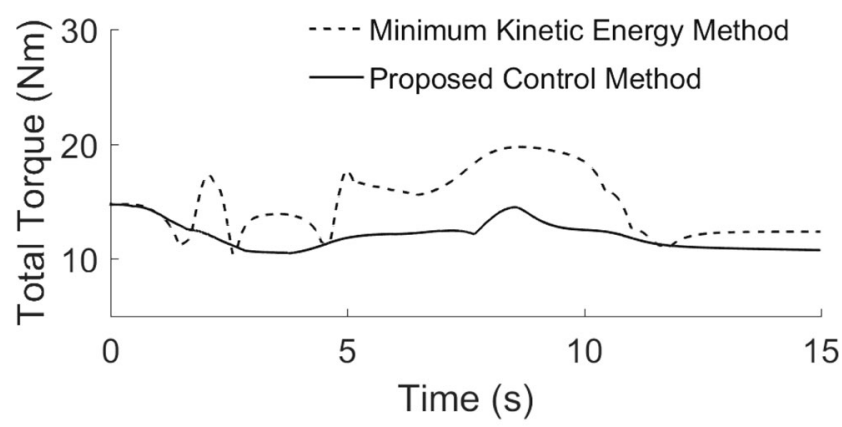

(a) Torque for a particular starting condition.

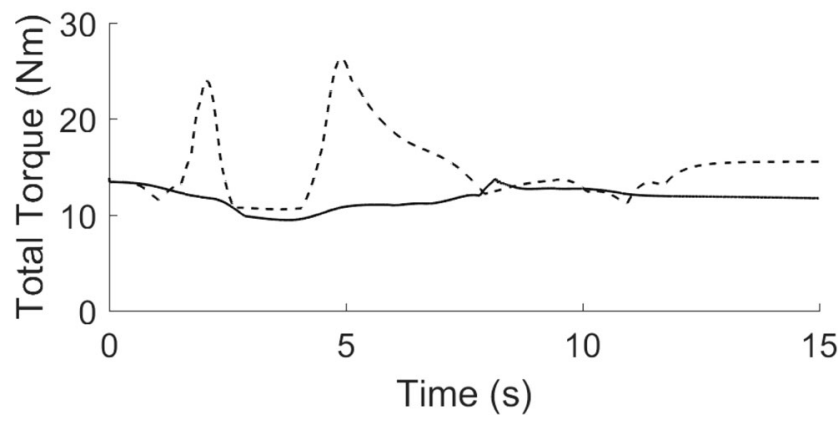

(b) Torque with another starting condition.

Fig. 7 Sum of absolute joint torques for the blasting simulation, starting from two different inital joint states. Notice that the MKE method increased the overall joint torque when returning to the same end-effector pose (b). The proposed control method decreased the overall joint torque for both

Then from Eqs. 10, 51, and 52:

$$
\begin{gathered}
\mathbf{K}_{\mathrm{q}} \Delta \mathbf{q}=\mathbf{J}^{\mathrm{T}} \Delta \boldsymbol{v} \\
\mathbf{K}_{\mathrm{q}} \Delta \mathbf{q}=\mathbf{J}^{\mathrm{T}} \mathbf{K}_{\mathrm{c}} \Delta \mathbf{x} \\
\mathbf{K}_{\mathrm{q}}=\mathbf{J}^{\mathrm{T}} \mathbf{K}_{\mathrm{c}} \mathbf{J} .
\end{gathered}
$$

Equation 55 can be used to solve the necessary joint configuration/stiffness given $\mathbf{K}_{\mathrm{c}}$. It can be seen from Eq. 42 that:

$\mathbf{K}_{\mathrm{q}}=\partial \boldsymbol{\tau}_{\mathrm{R}} / \partial \mathbf{q}$,

and by re-examining the cost function (37) used to optimize the joint torques:

$\nabla h(\mathbf{q})=\mathbf{K}_{\mathrm{q}}^{\mathrm{T}} \mathbf{G} \mathbf{J}^{\mathrm{T}} \boldsymbol{v}$.

In Eq. $57, \mathbf{J}^{\mathrm{T}}$ maps the wrench $\boldsymbol{v}$ from the task space to the joint space. The matrix $\mathbf{G}$ weights the torques, and the result is modulated by the joint stiffness $\mathbf{K}_{\mathrm{q}}$. That is, the redundant task will move the manipulator to increase the joint stiffness against the wrench. The Cartesian stiffness control and the torque minimization method presented here both involve the optimization of the joint stiffness. Therefore, the control method proposed in this paper could have the potential to achieve similar objectives. This may warrant further research. 
Fig. 8 Joint torques and joint angles for the robotic blasting simulation. The proposed control method is able to simultaneously reduce the joint torque required for the task, and avoid joint limits
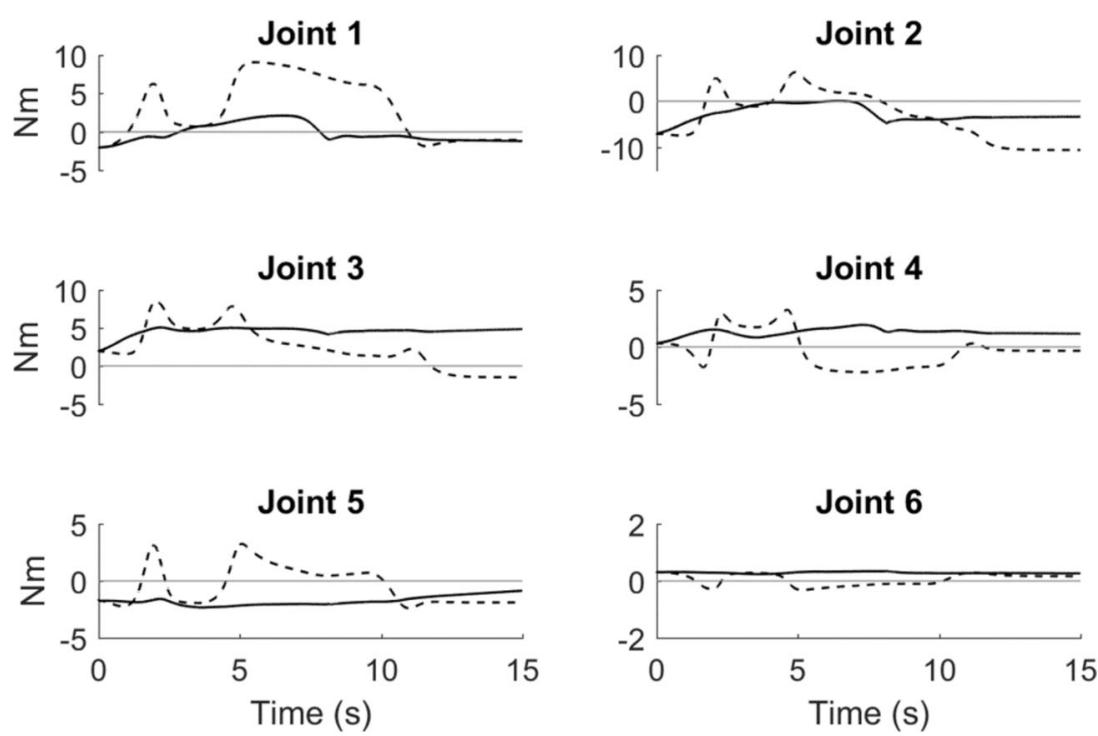

(a) Plot of individual joint torques.
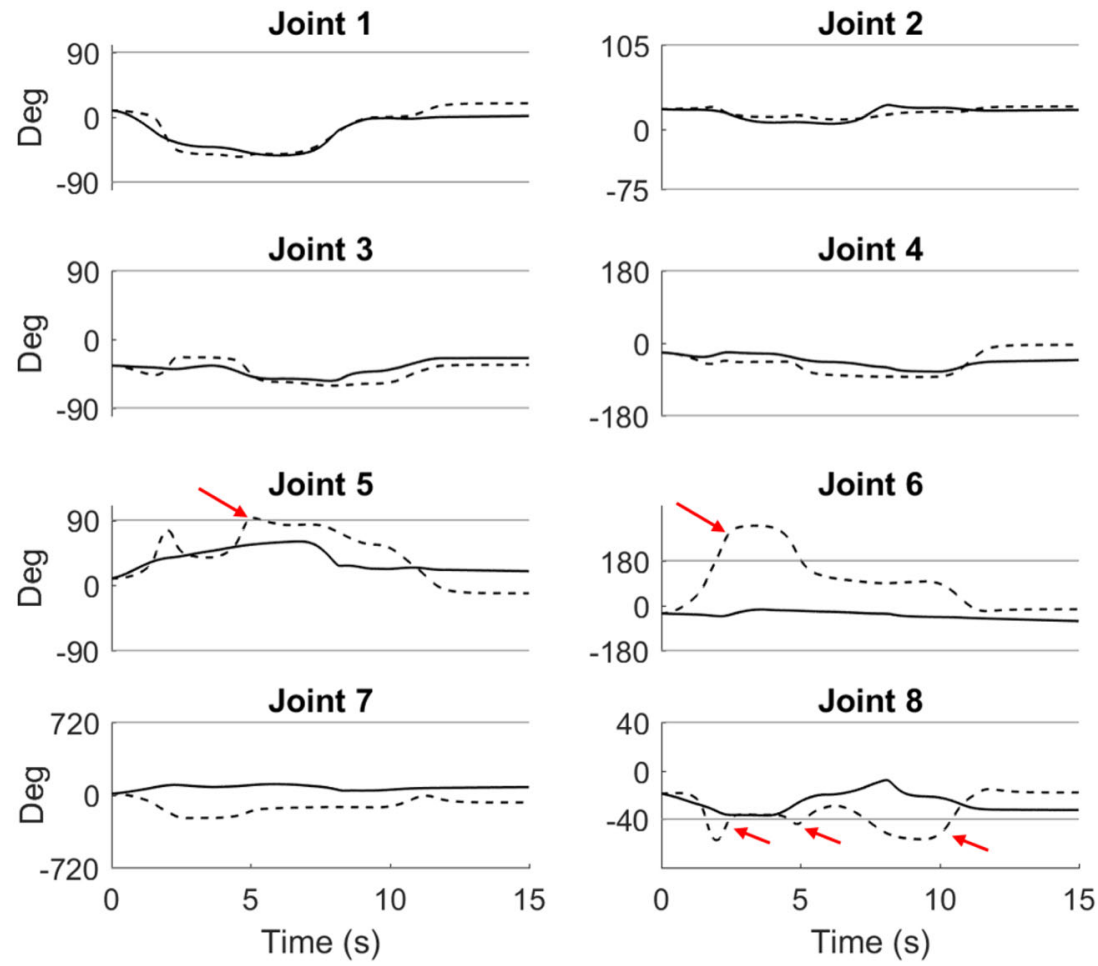

(b) Plot of individual joint angles.

\section{Conclusion}

Robotic manipulators are often required to undertake demanding tasks in which they must contend with various forces acting on the end-effector. The design selection of a manipulator is a careful balance of properties such as dexterity, workspace volume, degrees of freedom, and payload capacity. Given this, a manipulator may have to work at or above its payload capacity given constraints imposed on other properties due to the task requirements. In such a case, it is imperative that the joint configuration is carefully controlled to reduce joint torque.

Most methods in literature for joint torque minimization of redundant manipulators are formulated with respect to the internal dynamics of the system. However, when external forces are applied to the end-effector, these methods may 
increase the overall joint torque. To address this issue, a method was proposed in this paper to minimize the overall joint torque where an external loading on the end-effector must be accounted for. This was applied through null space control, such that the joints can autonomously rearrange themselves and reduce the effects of the external force without affecting the end-effector. Furthermore, the proposed method factors in weighting on the joint motion which can be used to incorporate physical constraints on the solution.

Two case studies were considered to verify the proposed control method. In the first scenario, a manipulator was simulated lifting and moving a heavy object. In the second case study, a manipulator was simulated performing highpressure blasting, where fluid reaction forces push back on the end-effector. Using the proposed control method, the overall joint torques were reduced compared to conventional methods. Furthermore, there is the added potential that a manipulator may work beyond its nominal payload capacity using this control method.

Acknowledgements Research supported in part by the Australian Research Council (ARC) Linkage Project (LP150100935) and the Roads and Maritime Services NSW.

Open Access This article is distributed under the terms of the Creative Commons Attribution 4.0 International License (http:// creativecommons.org/licenses/by/4.0/), which permits unrestricted use, distribution, and reproduction in any medium, provided you give appropriate credit to the original author(s) and the source, provide a link to the Creative Commons license, and indicate if changes were made.

Publisher's Note Springer Nature remains neutral with regard to jurisdictional claims in published maps and institutional affiliations.

\section{References}

1. Bhangale, P., Agrawal, V., Saha, S.: Attribute based specification, comparison and selection of a robot. Proceedings of the 11th National Conference on Machines and Mechanisms, pp. 131-138 (2003)

2. Yoshikawa, T.: Analysis and control of robot manipulators with redundancy. Robotics Research: The First International Symposium, Cambridge, pp. 735-747 (1984)

3. Liu, D.K., Dissayanake, G., Manamperi, P., Brooks, P., Paul, G., Webb, S., Kirchner, N., Chotiprayanakul, P., Kwok, N.M., Ren, T.: A robotic system for steel bridge maintenance: Research challenges and system design, Proceedings of the Australasian Conference on Robotics and Automation (ACRA) (2008)

4. Denso Wave Incorporated, 6-Axis robots VM series specifications (Online), (http://www.denso-wave.com/en/robot/product/five-six/ vm__Spec.html) Accessed 12th Feb 2018

5. Paul, G., Webb, S.S., Liu, D., Dissanayake, G.: A robotic system for steel bridge maintenance: Field testing, Proceedings of the Australasian Conference on Robotics and Automation (ACRA) (2010)
6. Manamperi, P., Brooke, P., Kaluarachchi, W., Peters, G., Ho, A., Lie, S., To, A., Payl, G., Rushton-Smith, D., Webb, S., Liu, D.K.: Robotic Grit-blasting: Engineering Challenges. Sustainable Bridges: The Thread of Society, pp. 321-330 (2011)

7. Liu, D.K., Dissanayake, G., Miro, J., Waldron, K.: Infrastructure robotics: Research challenges and opportunities. Int. Symp. Autom. Robot. Constr. 31, 1 (2014)

8. Kirchner, N., Paul, G., Liu, D.K.: Bridge maintenance robotic arm: mechanical technique to reduce the nozzle force of a sandblasting rig. J. Wuhan Univ. Technol. 28(Suppl. 2006-083), 12-18 (2006)

9. Woolfrey, J., Liu, D.K., Carmichael, M.: Kinematic control of an autonomous underwater Vehicle-Manipulator system using autoregressive predictive of vehicle motion and model predictive control. IEEE International Conference on Robotics and Automation (ICRA), pp. 4591-4596 (2016)

10. Hollerbach, J., Suh, K.: Redundancy resolution of manipulators through torque optimization. IEEE J. Robot. Autom. 3(4), 308316 (1987)

11. Nedungadi, A., Kazerouinian, K.: A local solution with global characteristics for the joint torque optimization of a redundant manipulator. Adv. Robot. 6(5), 631-645 (1989)

12. Kang, H., Freeman, R.: Joint torque optimization of redundant manipulators via the null space damping method. IEEE International Conference on Robotics and Automation, pp. 520-525 (1992)

13. Chung, C., Lee, B., Kim, M., Lee, C.: Torque optimizing control with singularity-robustness for kinematically redundant robots. J. Intell. Robot. Syst. 28(3), 231-258 (2000)

14. Tang, W., Wang, J.: Two recurrent neural networks for local joint torque optimization of kinematically redundant manipulators. IEEE Trans. Syst. Man. Cybern. Part B (Cybern.) 30(1), 120-128 (2000)

15. Zhang, Y., Ge, S., Lee, T.: A unified quadratic-programmingbased dynamical system approach to joint torque optimization of physically constrained redundant manipulators. IEEE Trans. Syst. Man. Cybern. Part B (Cybern.) 34(5), 2126-2132 (2004)

16. Zhang, Y., Guo, D., Ma, S.: Different-level simultaneous minimization of joint-velocity and joint-torque for redundant robot manipulators. J. Intell. Robot. Syst. 72(3-4), 301-324 (2013)

17. Flacco, F., De Luca, A.: Discrete-time redundancy resolution at the velocity level with acceleration/torque optimization properties. Robot. Auton. Syst. 70, 191-201 (2015)

18. Bussmann, K., Dietrich, A., Ott, C.: Whole-Body Impedance control for a planetary rover with robotic arm: theory, Control Design, and Experimental Validation. IEEE International Conference on Robotics and Automation (ICRA), pp. 910-917 (2018)

19. Luo, L., Yuan, C., Yan, R.J., Yuan, Q., Wu, J., Shin, K.S.: Trajectory planning for energy minimization of robotic manipulators using the lagrange interpolation method. Int. J. Precis. Eng. Manuf. 16(5), 911-917 (2015)

20. Chan, T., Dubey, R.: A weighted least-norm solution based scheme for avoiding joint limits for redundant joint manipulators. IEEE Trans. Robot. Autom. 11(2), 286-292 (1995)

21. Dariush, B., Hammam, G.B., Orin, D..: Constrained Resolved Acceleration Control for Humanoid Robots. IEEE/RSJ International Conference on Intelligent Robots and Systems, pp. 710-717 (2010)

22. Chen, P., Shan, C., Xiang, J., Wei, W.: Moving Obstacle Avoidance for Redundant Manipulator via Weighted Least Norm Method, 27th Chinese Control and Decision Conference (CCDC), pp. 6181-6186 (2015)

23. Wampler, C.: Manipulator inverse kinematic solutions based on vector formulations and Damped-Least-Sqaures methods. IEEE Tran. Syst. Man. Cybern. SMC-16 1, 93-101 (1986) 
24. Chiaverini, S., Egeland, O., Kanestrom, R.: Achieving userdefined accuracy with damped least-squares inverse kinematics. Fifth International Conference on Advanced Robotics, pp. 672677 (1991)

25. Hsu, P., Hauser, J., Sastry, S.: Dynamic control of redundant manipulators. J. Robot. Syst. 6(2), 133-148 (1989)

26. Rethinkrobotics.com, Sawyer Technical Specifications, (Online) (http://www.rethinkrobotics.com/sawyer/tech-specs/), Accessed 5th Feb 2018

27. Igus.com, Robolink WR, (Online), (https://www.igus.com/wpck/ 16962/N16_10_11), Accessed 5th Feb 2018

28. Choi, H.R., Chung, W.K., Youm, Y.: Stiffness analysis and control of redundant manipulators IEEE. International Conference on Robotics and Automation (ICRA), pp. 689-695 (1994)

29. Ang, M.H., Andeen, G.B.: Specifying and achieving passive compliance based on manipulator structure. IEEE Trans. Robot. Autom. 11(4), 504-515 (1995)

30. Li, Y., Kao, L.: Stiffness control on redundant manipulators: a unique and kinematically consistent solution. IEEE International Conference on Robotics and Automation (ICRA), pp. 3956-3961 (2004)

31. Ajoudani, A., Tsagarakis, N.G., Bicchi, A., Ajoudani, A., Tsagarakis, N.G.: Bicchi, On the role of robot configuration in Cartesian stiffness control", IEEE International Conference on Robotics and Automation (ICRA), pp. 1010-1016 (2015)

32. Gasparri, G.M., Gabiani, F., Garabini, M., Pallottino, L., Catalon, M., Grioli, G., Perischin, R., Bicchi, A.: Robust optimization of system compliance for physical interaction in uncertain scenarios. IEEE-RAS 16th International Conference on Humanoid Robots (Humanoids), pp. 911-918 (2016)
Jon Woolfrey received his B.E. in Mechanical and Mechatronic Engineering from the University of Technology Sydney (UTS) in 2015. He is currently a Doctoral Candidate with UTS, focusing on controlling mobile manipulators under disturbance and working with heavy loads. His research interests include control of robotic systems, optimization, statistical modelling, and design. He has helped develop mobile robots for infrastructure maintenance and control methods to enable them to operate effectively.

Wenjie Lu received his B.S. in Mechatronic Engineering from Zhejiang University, Hangzhou, China in 2009. He then received his M.S. and Ph.D. in Mechanical Engineering from Duke University, Durham, NC, USA in 2011 and 2014 respectively. His research interests include the learning of efficient motion planners and controllers for mobile robots that are subject to uncertainties in 3D complex environment. He has been developing such algorithms via integrating machine learning, approximate dynamic programming, hybrid systems, information theories, and nonparametric Bayesian models.

Dikai Liu received his Ph.D. degree in 1997. His main research interest is robotics including exploration, motion planning, robot teams, and physical human-robot interaction. He has been developing novel methods and algorithms that enable robots to operate in unstructured and complex 3D environments autonomously or collaboratively with human users. Example robotic systems he developed and practically deployed include autonomous grit-blasting robots for steel bridge maintenance, assistive robots for human strength augmentation in industrial applications and bioinspired climbing robots for steel structure inspection. 